\title{
Coherent Multiphoton Control of Gallium Phosphide Nanodisk Resonances
}

\author{
Vikas Remesh ${ }^{1}$, Gustavo Grinblat ${ }^{2,3}, \mathrm{Yi} \mathrm{Li}^{2,4}$, Stefan A. Maier ${ }^{2,4}$ and Niek F. van Hulst ${ }^{1,5}$ \\ 1. ICFO- Institut de Ciences Fotoniques, The Barcelona Institute of Science and Technology, 08860 Castelldefels (Barcelona), \\ Spain \\ 2. The Blackett Laboratory, Department of Physics, Imperial College London, London SW7 2AZ, United Kingdom \\ 3. Departamento de Física, FCEN, IFIBA-CONICET, Universidad de Buenos Aires, C1428EGA Buenos Aires, Argentina \\ 4. Chair in Hybrid Nanosystems, Nanoinstitute Munich, Ludwig-Maximilians-Universität Munich, 80539 Munich, Germany \\ 5. ICREA- Institució Catalana de Recerca i Estudis Avançats, Barcelona 08010, Spain
}

\begin{abstract}
High-index dielectric nanoantennas have recently emerged as promising alternatives to plasmonic nanoantennas for concentrating and manipulating light at the nanoscale. For example, Gallium Phosphide $(\mathrm{GaP})$ nanoantennas display extremely low losses in the visible range, high nonlinearities, support both electric and magnetic resonances, and are nicely compatible with CMOS fabrication technology. Especially, the low losses and large nonlinearities are promising for ultrafast optical switching and truly all-optical control of GaP nanodevices. Here first we use two- and three-photon excitation of GaP nanodisks to probe the size-dependent resonance enhancement of second harmonic and bandgap emission. Next, we show, by spectral phase control of broadband pulsed excitation, that GaP nanoantennas outperform their metal counterparts in supporting nonlinear optical coherences.
\end{abstract}

Keywords: nanoantennas, optical nonlinearity, gallium phosphide, femtosecond pulse shaping, multiphoton luminescence, coherent control.

Plasmonic nanoantennas have been widely used for concentrating and localizing electromagnetic energy to enhance fluorescence and in Raman spectroscopies, ${ }^{1,2}$ controlling the directionality of radiative emission, ${ }^{3,4}$ among others. Most of the exciting features of such systems result from collective electronic oscillations i.e. localized surface plasmons (LSPs). However, due to the lossy nature and short dephasing times of LSPs, the coherent control of nanoscale nonlinear optical excitations in metal nanoantennas has been limited to ultrashort pulses below $30 \mathrm{fs}^{5}$ 
In the past few years, all-dielectric nanoantennas with high refractive indices, like $\mathrm{Si}$, Ge or combinations of III-V materials such as gallium arsenide (GaAs), aluminum gallium arsenide $(\mathrm{AlGaAs})$ or gallium phosphide $(\mathrm{GaP}),{ }^{6-10}$ have attracted great attention as alternative candidates for metal nanoantennas due to their ultra-low losses and compatibility with semiconductor processing technologies. These nanoantennas support optically-induced Mie resonances in the visible and near-infrared range, allowing wide wavelength tunability. ${ }^{6}$ In addition, unlike their metallic counterparts, they can support both strong electric and magnetic modes, bringing up unique optical properties. ${ }^{11,12}$ Furthermore, they allow for high optical field enhancement, ${ }^{10,13}$ and spectral and directional shaping of emission. ${ }^{8,9}$

Dielectric nanoantennas with high second- and third-order nonlinear susceptibilities are also useful in enhancing and manipulating various nonlinear optical processes, like two-photon absorption (TPA), second harmonic generation (SHG), third harmonic generation (THG), optical Kerr effect (OKE) and nonlinear mixing.,10,14,15 Their strong intrinsic nonlinear response, aided by field enhancement when excited resonantly, make these materials ideal for nonlinear optical coherent control experiments. In this Letter, we explore the performance of single $\mathrm{GaP}$ nanodisk antennas when excited with ultrashort pulses of variable pulse duration and controlled spectral phase. We first analyze the emission spectrum of GaP nanodisks to find the presence of SHG and bandgap emission, dominated by 3-photon absorption at sufficiently large pump power, and observe that both signals peak at specific disk diameters due to Mie resonances. For an appropriately defined coherent contrast, we then demonstrate that the most efficient nonlinear nanodisk delivers a phase control contrast of up to 70-80\% at the bandgap emission, much stronger than the SHG contrast, and which largely outlasts the coherent response produced by the 2-photon luminescence of a reference Au nanoantenna.

Arrays of GaP nanodisks of $210 \mathrm{~nm}$ height and diameters (D) varying from $80 \mathrm{~nm}$ to $560 \mathrm{~nm}$ were fabricated by patterning a $350 \mu \mathrm{m}$-thick crystalline $\mathrm{GaP}(100)$ wafer via e-beam 
lithography, followed by an etching procedure as described elsewhere. ${ }^{10} \mathrm{~A} 4 \mu \mathrm{m}$ center-tocenter pitch was chosen to prevent optical coupling between adjacent resonators. The nanoantennas were excited with a broadband Titanium:Sapphire laser (Octavius 85M, Menlo Systems) tuned to a central wavelength of $790 \mathrm{~nm}$ with $\sim 100 \mathrm{~nm}$ bandwidth, corresponding to 14 fs Fourier limited pulse duration. The laser pulses were focused onto individual nanodisks by a 0.95 numerical aperture, 100x air-objective from the air side. The sample was placed on a $\mathrm{XYZ}$ piezo stage in a home-built microscope, and the nonlinear signal from single nanodisks was detected in reflection geometry using an avalanche photodiode (APD) or a spectrometer coupled to an emCCD camera (Andor). A 4f shaper in folded geometry equipped with a spatial light modulator (640 pixels, CRi, adapted from MIIPS box, Biophotonics Solutions Inc.) enabled phase-amplitude shaping of the excitation pulses as described in the literature. ${ }^{15}$

First, to study the nonlinear response of the nanodisks, we evaluated their emission spectrum in the visible range by pumping at different average excitation intensity up to 5 $\mathrm{GW} / \mathrm{cm}^{2}$, with linearly polarized light. Figure 1a shows the emission spectrum of a nanodisk. Two distinct regions can be noticed: SHG emission centered at $395 \mathrm{~nm}$, and a $30 \mathrm{~nm}$-wide luminescence band centered at $550 \mathrm{~nm}$, which we attribute to excitonic emission as it corresponds to $\mathrm{GaP}$ bandgap wavelength. Figure $1 \mathrm{~b}$ shows the evolution of the nonlinear spectrum with power. The experimental data was fitted with a power law $\propto I^{m}$, where $I$ the incident power and $m$ the exponent in the power dependence. Figure 1a shows the fitted power exponent $m$ at the different emission wavelengths, revealing that SHG obeys a quadratic power law $(m=2)$, as expected, while the $550 \mathrm{~nm}$ band demonstrates a cubic power dependence $(m$ $=3$ ), indicating that the excitonic emission is dominated by 3-photon absorption. 


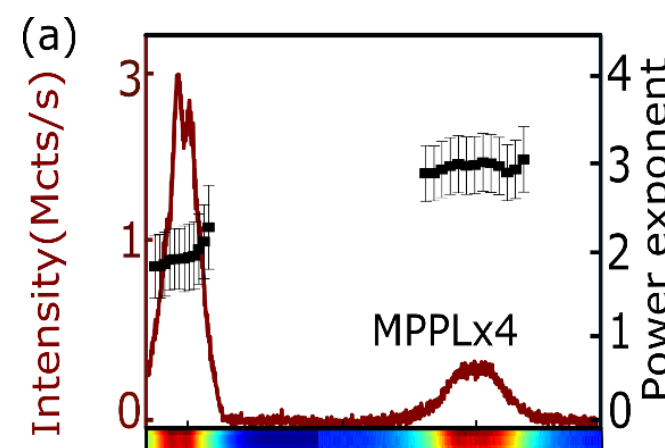

Figure 1. Multiphoton response

(a) Nonlinear emission spectrum of a $440 \mathrm{~nm}$ diameter GaP nanodisk, and exponent of power law fits at different wavelengths.

(b) Evolution of GaP nanodisk SHG and bandgap emission spectrum with the excitation power. SHG data was acquired for excitation powers up to $1 \mathrm{GW} / \mathrm{cm}^{2}$ and normalized to bandgap emission power levels to enable comparison.

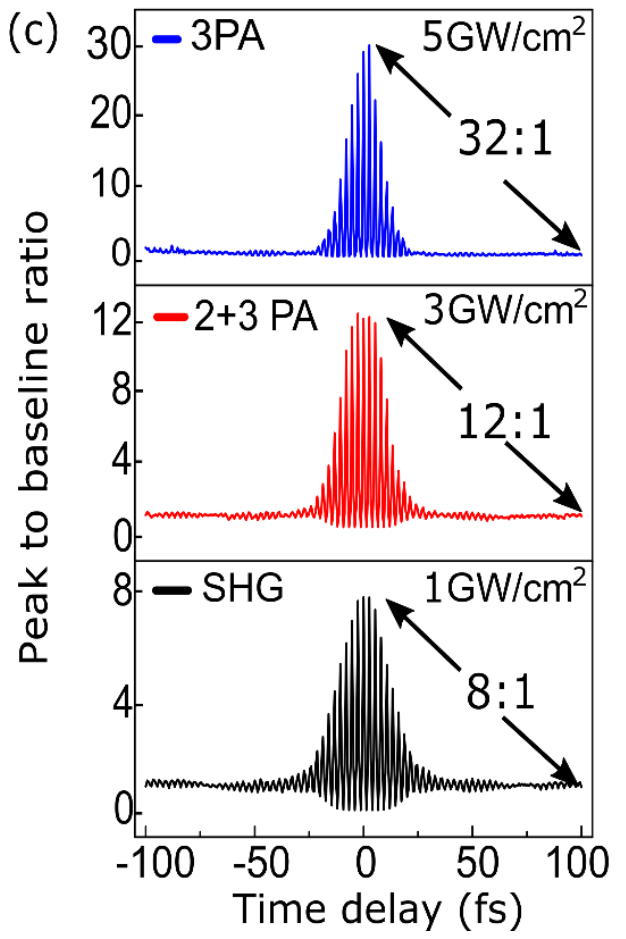

(c) iFROG scans for the different nonlinear signals. SHG (black curve) demonstrates a second order process. Bandgap emission reveals a $2+3$ photon absorption effect at low powers (red curve), that becomes a pure 3photon absorption process at high powers (blue curve).

To confirm the nonlinear nature of the excitonic component of the spectrum, we performed interferometric frequency-resolved optical gating (iFROG) scans at different excitation power levels. In an interferometric intensity autocorrelation measurement, the ratio $r$, of the intensity at zero delay to the baseline intensity at infinite delay, is related to the order $m$ of the multiphoton process as ${ }^{16} \quad r=2^{2 m-1}$. 
The obtained iFROG spectrograms, integrated over the excitonic emission range, as shown in Figure 1c, indicate that both 2 and 3 photon processes coexist at low excitation powers (3 $\mathrm{GW} / \mathrm{cm}^{2}$, red curve), while a 3-photon process governs $(r=32: 1)$ when the power is increased ( $5 \mathrm{GW} / \mathrm{cm}^{2}$, blue curve), i.e. multiphoton photoluminescence (MPPL). Black data in the graph corresponds to SHG, displaying the expected second order response $(r=8: 1)$.

Next, we analyzed the influence of the GaP nanodisk size and corresponding Mie resonances on the SHG and MPPL emissions. To that end, nonlinear raster scan images of 5x5 arrays of $\mathrm{GaP}$ nanodisks of increasing diameter $(80$ to $560 \mathrm{~nm}$ ) were attained, exciting the sample at average excitation intensity of $1 \mathrm{GW} / \mathrm{cm}^{2}$ for SHG and $5 \mathrm{GW} / \mathrm{cm}^{2}$ for MPPL. These power levels enabled the study of 2 (SHG) and 3 (high-power MPPL) photon processes, with sufficient signal-to-noise ratio, while larger excitation powers for either nonlinear process would have saturated the APD. Dichroic filters were used to isolate either the SHG signal or the MPPL contribution. Figure 2a shows, from left to right, a scanning electron microscope (SEM) image of a 5x5 disk array with corresponding registered SHG and MPPL images. The dependence of the emission intensities on the disk diameter, as extracted from the maximum count values in the nonlinear scans, is depicted in Figure 2b. Both SHG and MPPL response present relative maxima at specific diameter values. To establish the origin of this effect, we performed numerical simulations of the scattering cross section and near-field distribution of the nanoantennas, using the commercial software Lumerical. The complex refractive index of $\mathrm{GaP}$ was determined from measured ellipsometry data and used as an input for the numerical calculations. The inset of Figure $2 \mathrm{c}$ exhibits the calculated electric field intensity distribution for $\mathrm{D}=450 \mathrm{~nm}$ at the scattering maximum, which occurs around the pump wavelength used in the experiments, as shown in the main graph. The electric field is confined to the top region of the nanodisk, with a field enhancement factor with respect to the incident field of $\sim 6$. 

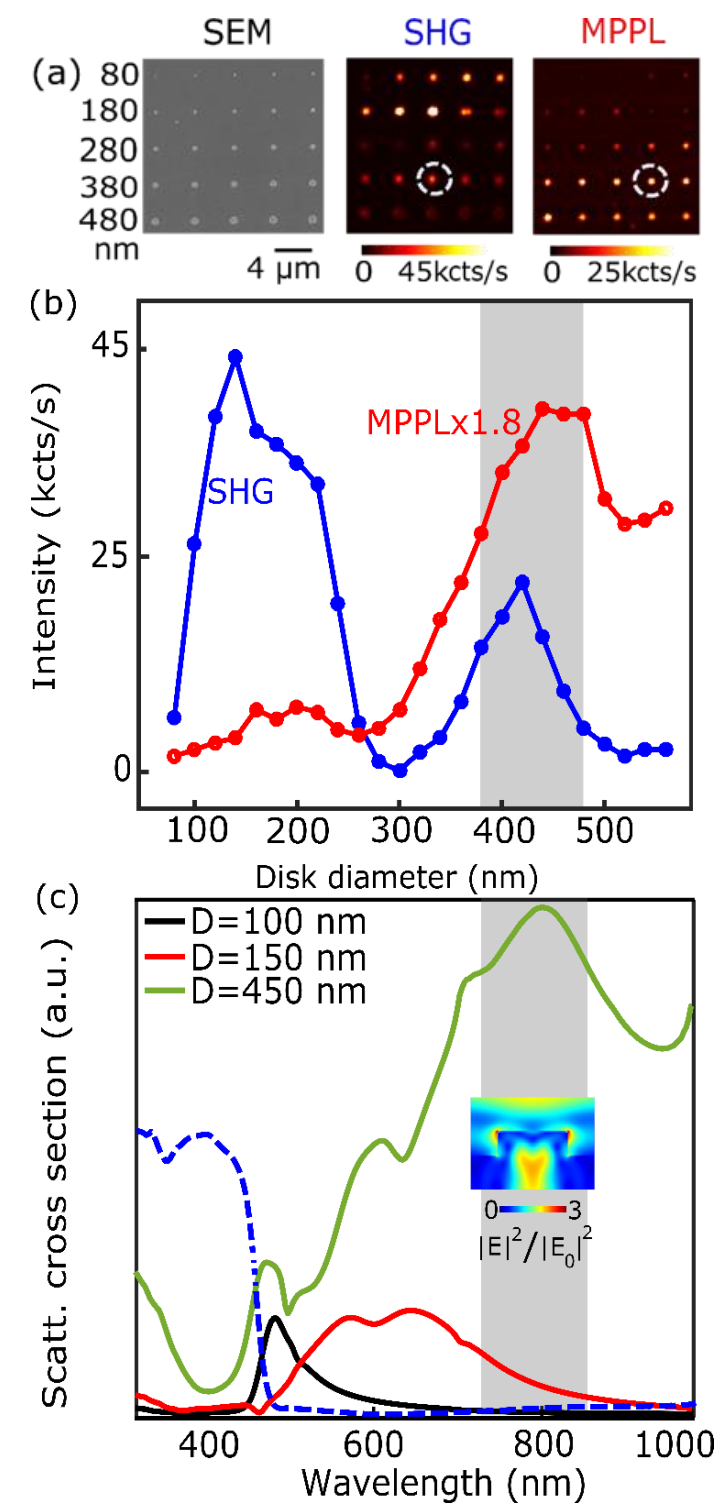

Figure 2. . Geometrical resonances. (a) SEM image of a $5 \times 5$ array of GaP nanodisk antennas (left panel) and corresponding SHG (middle) and MPPL (right) images. The disk diameters increase from $80 \mathrm{~nm}$ to $560 \mathrm{~nm}$ in steps of $20 \mathrm{~nm}$. The resonant nanodisks for the experiment are encircled. (b) Measured SHG and MPPL intensities as a function of disk diameter. (c) Simulated wavelength dependence of the scattering cross section of GaP nanodisks of different diameters. The dashed blue curve shows the simulated absorption cross section of $D=450 \mathrm{~nm}$ disk. The inset shows the simulated field intensity distribution for $D=450 \mathrm{~nm}$, in the plane of the excitation polarization, excited from airside at $800 \mathrm{~nm}$ wavelength resonance. The grey shaded area represents the wavelength region of the excitation laser, which resonates with disk sizes around $440 \mathrm{~nm}$ diameter, as highlighted in (b). 
Given that SHG and 3-photon absorption photoluminescence scale with the fourth and sixth power of the incident electric field amplitude, respectively, a strong increase in the signals is expected when the fundamental wavelength is at resonance, explaining the observed peaks at $\mathrm{D} \sim 440 \mathrm{~nm}$ in Figure 2b. The additional measured maxima at around $\mathrm{D}=100 \mathrm{~nm}$ for SHG and $\mathrm{D}=200 \mathrm{~nm}$ for MPPL would arise from resonances at the emission wavelengths, as indicated by numerical calculations for disks of smaller sizes. We attribute differences between experimental and simulated resonant wavelengths and diameter values to imperfections in the fabrication ${ }^{7,10,18,19}$ process especially for disks of the smallest diameters. Figure $2 b$ also reveals that small resonant diameters outperform large resonant sizes for SHG, while the opposite occurs for MPPL. This can be explained by the broad scattering resonance for $\mathrm{D}=440 \mathrm{~nm}$ in Figure 2c which also covers the $550 \mathrm{~nm}$ band (but not the SHG wavelength), giving rise to a doubly resonant effect that further boosts the MPPL emission, as has been observed in nonlinear nanoantennas with resonances at both the excitation and emission wavelengths. ${ }^{20-26}$ In addition, since the resonance at the fundamental wavelength has a stronger influence on the MPPL process, being of higher order than SHG, a particularly large reduction in the MPPL emission intensity is expected at the smallest diameters, where the resonance at the fundamental wavelength is absent. We also note that the absorption cross section (shown as dashed blue curve in Figure 2c) does not change much with the disk sizes due to substrate effect. Thus, further simulation studies considering the emission patterns of the nonlinear signals, as well as the surface and volume contributions to the nonlinear responses, would be needed to quantitatively describe the experimental findings.

We turn now our attention toward the phase control experiments, which were performed on the nanodisk of 420-460 nm diameter, as they deliver both efficient SHG and MPPL. Firstly, we measured the temporal response of the nonlinear signals when chirping the excitation beam. The spectral phase $\varphi$ of a laser pulse is generally described as a Taylor expansion with respect 
to its central frequency $\omega_{0}(790 \mathrm{~nm}$ wavelength in our measurements), in which the second order phase term $\varphi_{2}=\frac{1}{2} \varphi^{(2)}\left(\omega-\omega_{0}\right)^{2}$ determines the chirp. Modifying the group delay dispersion (GDD), $\varphi^{(2)}$, changes the pulse duration from its Fourier limit $(\tau)$ to a desired value $\left(\tau_{\text {out }}\right)$. For a Gaussian laser pulse, $\varphi^{(2)}$ and the pulse duration are related by:

$$
\tau_{\text {out }}^{2}=\tau^{2}+\left(4 \ln 2 \frac{\varphi^{(2)}}{\tau}\right)^{2}
$$

In this experiment, we varied $\varphi^{(2)}$ from $-1000 \mathrm{fs}^{2}$ to $1000 \mathrm{fs}^{2}$. In time domain, this corresponds to stretching the pulse from 14 fs to 190 fs. It is important to note that positive and negative $\varphi^{(2)}$ values imply the same result in time domain. The obtained multiphoton phase spectrogram, averaged over 400 scans, is shown in the top and bottom left panels of Figure 3a, demonstrating that the SHG spectral intensity falls off slower than the MPPL signal. Next, in addition to $\varphi^{(2)}$, we added a static $\pi$ phase step at a frequency $\omega_{\text {step }}$ in the laser spectrum, defined as $\varphi_{\pi}=\frac{\pi}{2} \operatorname{sign}\left(\omega-\omega_{\text {step }}\right)$, where $\operatorname{sign}\left(\omega-\omega_{\text {step }}\right)$ jumps from -1 to +1 at $\omega_{\text {step }}$. A static $\pi$ phase step has earlier been reported as an effective phase control method to manipulate the interfering pathways in a multiphoton process. ${ }^{27,28}$ The top and bottom right panels of Figure 3a show the result of applying $\varphi^{(2)}+\varphi_{\pi}$ to the laser pulses at $\omega_{\text {step }}=\omega_{0}$. A wider response in phase is now observed in the SHG and MPPL scans. To better analyze these results, it is convenient to define a coherent contrast $(\beta)$ as: ${ }^{5}$

$$
\beta=\frac{{ }^{I}{ }^{(2)}-I_{\varphi^{(2)}}+\varphi_{\pi}}{I_{\varphi}(2)}
$$

where $I_{\varphi^{(2)}}$ and $I_{\varphi^{(2)}+\varphi_{\pi}}$ correspond to integrated intensities over the emission spectrum that can be directly computed from the GDD scans in Figure 3a. Figure $3 b$ shows the obtained plots of $\beta$ against $\varphi^{(2)}$. When $\varphi^{(2)}=0 \mathrm{fs}^{2}$, the contrast for MPPL $\left(\beta_{M P P L}\right)$ is $\sim 80 \%$, which is a factor of 1.4 higher than that for SHG $\left(\beta_{S H G} \sim 56 \%\right)$. The theoretical SHG response is shown in black solid line, as calculated from the laser spectrum using standard nonlinear theory from 
the literature. ${ }^{5}$ As $\varphi^{(2)}$ is increased, $\beta_{M P P L}$ drops and crosses the zero level at $330 \mathrm{fs}^{2}$, while $\beta_{S H G}$ is found to fall faster, crossing the zero level at $250 \mathrm{fs}^{2}$, flipping sign to remain constant at approximately $-20 \%$. Remarkably, for MPPL, when $\varphi^{(2)}$ is further increased, its corresponding $\beta$ value grows further in the negative direction reaching $-70 \%$ at $\sim 500 \mathrm{fs}^{2}$, highly surpassing the performance of SHG.

(a)

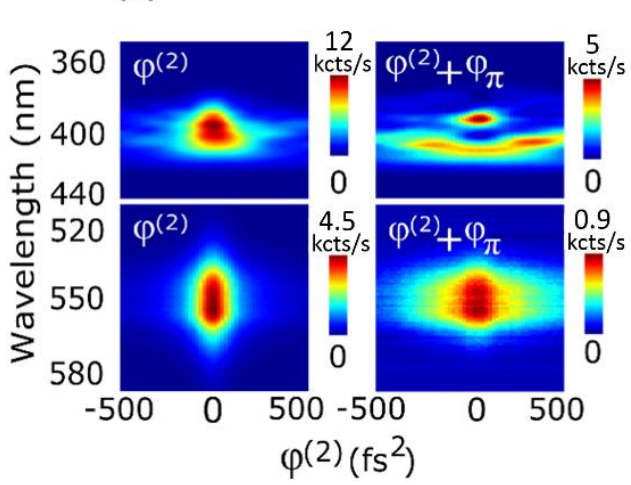

(b) Pulse duration (fs)

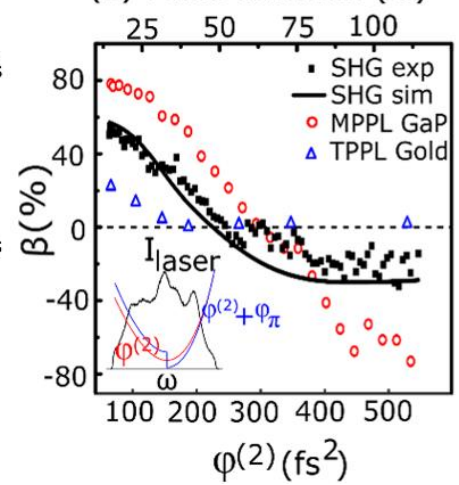

(c)

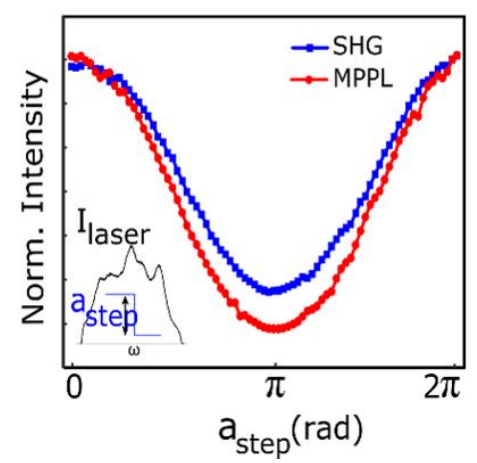

Figure 3. Phase control (a) GDD scans for SHG and MPPL without (left panels) and with (right panels) an additional $\pi$ step. (b) Coherent contrast $\beta$ of SHG and MPPL on increasing chirp and pulse length of the excitation pulse. For comparison, also shown are TPPL results from a resonant Au nanorod (140 $\mathrm{nm}$ length, $50 \mathrm{~nm}$ width and height). The inset shows the spectral phase of a quadratic term $\varphi^{(2)}$ with and without a spectral $\pi$ phase step $\left(\varphi_{\pi}\right)$ on top. (c) Dependence of the nonlinear intensities on the phase step height $\left(a_{s t e p}\right)$ applied to the laser pulse a frequency $\omega_{0}$. For both SHG and the MPPL, the intensity minimum is achieved when the step height is $\pi$.

Clearly, the 3-photon absorption is much more sensitive to spectral change than the 2 photon counterpart. For reference, Figure $3 b$ also includes TPPL (two-photon absorption photoluminescence) results obtained from a resonant $\mathrm{Au}$ nanoantenna, which exhibits the poorest of contrasts and shortest of coherence times, as limited by the reduced dephasing times of LSPs.

Finally, to evaluate the strength of our phase step in controlling the multiphoton processes, we varied the amplitude of the phase step $\left(a_{\text {step }}\right)$ between 0 and $2 \pi$ at GDD $=0 \mathrm{fs}^{2}$ 
and studied its effect on the nonlinear signals. In all cases, the results showed a single minimum on a full sweep. Figure $3 \mathrm{c}$ shows the case of $\omega_{\text {step }}=\omega_{0}$, which reveals that both SHG and MPPL responses drop to a minimum for a step of phase amplitude $\pi$, proving the strength of the $\pi$ step in influencing the nonlinear processes. Again, the MPPL shows the strongest effect, yet for a 3-photon interaction, it is surprising to find the optimum at a phase difference of $\pi$.

In summary, we have studied the spectral phase control of nonlinear optical responses in GaP nanodisks at Mie resonances supporting efficient SHG and MPPL. By combining a dynamic GDD scan and a static $\pi$ phase step on a 14-fs excitation laser pulse, we determined the differences in the coherent responses between the MPPL and SHG processes. We showed that the coherent contrast for the MPPL effect is a factor of 1.4 (3.5) higher than that achieved for SHG at GDD $=0 \mathrm{fs}^{2}\left(\mathrm{GDD}>500 \mathrm{fs}^{2}\right)$. Notably, the coherency of the MPPL signal in the $\mathrm{GaP}$ nanodisk antenna outlasts that of the TPPL emission from its metal counterpart, demonstrating the potential of dielectric nanocavities for realizing coherent nonlinear nanodevices.

\section{Acknowledgements}

V.R. received support from the European Commission through the Erasmus Mundus Joint Doctorate Programme Europhotonics (Grant 159224-1-2009-1-FR-ERA MUNDUS-EMJD). G.G. acknowledges a Marie Skłodowska-Curie Fellowship. S.A.M. acknowledges the Nanosystems Initiative Munich Cluster of Excellence and the EPSRC program (EP/P033369/1). N.F.v.H. acknowledges the financial support by the European Commission (ERC Advanced Grant 670949-LightNet), Spanish Ministry of Economy ("Severo Ochoa" program for Centers of Excellence in R\&D SEV-2015-0522, Plan Nacional FIS2015-69258-P and Network FIS2016-81740-REDC), the Catalan AGAUR (2017SGR1369), Fundació Privada Cellex, Fundació Privada Mir-Puig, and Generalitat de Catalunya through the CERCA program. Authors thank Pawel Woźniak for fruitful discussions. 


\section{References}

(1) Novotny, L.; van Hulst, N. F. Antennas for Light. Nat. Photonics 2011, 5, 83-90.

(2) Kinkhabwala, A.; Yu, Z.; Fan, S.; Avlasevich, Y.; Müllen, K.; Moerner, W. E. Large SingleMolecule Fluorescence Enhancements Produced by a Bowtie Nanoantenna. Nat. Photonics 2009, 3, 654-657.

(3) Curto, A.G; Volpe T.H.; Kreuzer, M.P;Quidant, R.; van Hulst, N. F. Dot Coupled to a Nanoantenna. Science. 2010, 329, 930-933.

(4) Hancu, I. M.; Curto, A. G.; Castro-López, M.; Kuttge, M.; van Hulst, N. F. Multipolar Interference for Directed Light Emission. Nano Lett. 2014, 14, 166-171.

(5) Remesh, V.; Stührenberg, M.; Saemisch, L.; Accanto, N.; van Hulst, N. F. Phase Control of Plasmon Enhanced Two-Photon Photoluminescence in Resonant Gold Nanoantennas. Appl. Phys. Lett. 2018, 113, 211101.

(6) Kuznetsov, A. I.; Miroshnichenko, A. E.; Brongersma, M. L.; Kivshar, Y. S.; Luk'yanchuk, B. Optically Resonant Dielectric Nanostructures. Science. 2016, 354, aag2472.

(7) Grinblat, G.; Li, Y.; Nielsen, M. P.; Oulton, R. F.; Maier, S. A. Enhanced Third Harmonic Generation in Single Germanium Nanodisks Excited at the Anapole Mode. Nano Lett. 2016, 16, $4635-4640$.

(8) Staude, I.; Miroshnichenko, A. E.; Decker, M.; Fofang, N. T.; Liu, S.; Gonzales, E.; Dominguez, J.; Luk, T. S.; Neshev, D. N.; Brener, I.; Kivshar, Y. Tailoring Directional Scattering through Magnetic and Electric Resonances in Subwavelength Silicon Nanodisks. ACS Nano 2013, 7, $7824-7832$.

(9) Carletti, L.; Locatelli, A.; Neshev, D.; De Angelis, C. Shaping the Radiation Pattern of SecondHarmonic Generation from AlGaAs Dielectric Nanoantennas. ACS Photonics 2016, 3, 15001507.

(10) Cambiasso, J.; Grinblat, G.; Li, Y.; Rakovich, A.; Cortés, E.; Maier, S. A. Bridging the Gap between Dielectric Nanophotonics and the Visible Regime with Effectively Lossless Gallium Phosphide Antennas. Nano Lett. 2017, 17, 1219-1225.

(11) Yu, Y. F.; Zhu, A. Y.; Paniagua-Domínguez, R.; Fu, Y. H.; Luk’yanchuk, B.; Kuznetsov, A. I. High-Transmission Dielectric Metasurface with $2 \pi$ Phase Control at Visible Wavelengths. Laser Photonics Rev. 2015, 9, 412-418.

(12) Kuznetsov, A. I.; Miroshnichenko, A. E.; Fu, Y. H.; Zhang, J.; Luk'yanchukl, B. Magnetic Light. Sci. Rep. 2012, 2, 492.

(13) Regmi, R.; Berthelot, J.; Winkler, P. M.; Mivelle, M.; Proust, J.; Bedu, F.; Ozerov, I.; Begou, T.; Lumeau, J.; Rigneault, H.; Garcia-Parajo, M. F.; Bidault, S.; Wenger, J.; Bonod, N. All- 
Dielectric Silicon Nanogap Antennas to Enhance the Fluorescence of Single Molecules. Nano Lett. 2016, 16, 5143-5151.

(14) Carletti, L.; Locatelli, A.; Stepanenko, O.; Leo, G.; De Angelis, C. Enhanced Second-Harmonic Generation from Magnetic Resonance in AlGaAs Nanoantennas. Opt. Express 2015, 23, 2654426550.

(15) Grinblat, G.; Nielsen, M. P.; Dichtl, P.; Li, Y.; Oulton, R. F.; Maier, S. A. Ultrafast sub-30-fs all-optical switching based on gallium phosphide. Sci. Adv. 2019, 5, eaaw3262

(16) Accanto, N.; Nieder, J. B.; Piatkowski, L.; Castro-Lopez, M.; Pastorelli, F.; Brinks, D.; van Hulst, N. F. Phase Control of Femtosecond Pulses on the Nanoscale Using Second Harmonic Nanoparticles. Light Sci. Appl. 2014, 3, e143.

(17) Hyyti, J.; Perestjuk, M.; Mahler, F.; Grunwald, R.; Güell, F.; Gray, C.; McGlynn, E.; Steinmeyer, G. Field Enhancement of Multiphoton Induced Luminescence Processes in $\mathrm{ZnO}$ Nanorods. J. Phys. D. Appl. Phys. 2018, 51, 105306-105317.

(18) Liu, S.; Ihlefeld, J. F.; Dominguez, J.; Gonzales, E. F.; Eric Bower, J.; Bruce Burckel, D.; Sinclair, M. B.; Brener, I. Realization of Tellurium-Based All Dielectric Optical Metamaterials Using a Multi-Cycle Deposition-Etch Process. Appl. Phys. Lett. 2013, 102, 161905.

(19) Gholipour, B.; Adamo, G.; Cortecchia, D.; Krishnamoorthy, H. N. S.; Birowosuto, M. D.; Zheludev, N. I.; Soci, C. Organometallic Perovskite Metasurfaces. Adv. Mater. 2017, 29, 1604268 .

(20) Celebrano, M.; Wu, X.; Baselli, M.; Großmann, S.; Biagioni, P.; Locatelli, A.; De Angelis, C.; Cerullo, G.; Osellame, R.; Hecht, B.; Duò, L.; Ciccacci, F.; Finazzi, M. Mode Matching in Multiresonant Plasmonic Nanoantennas for Enhanced Second Harmonic Generation. Nat. Nanotechnol. 2015, 10, 412-417.

(21) Weber, N.; Protte, M.; Walter, F.; Georgi, P.; Zentgraf, T.; Meier, C. Double Resonant Plasmonic Nanoantennas for Efficient Second Harmonic Generation in Zinc Oxide. Phys. Rev. B 2017, 95, 205307.

(22) Aouani, H.; Navarro-Cia, M.; Rahmani, M.; Sidiropoulos, T. P. H.; Hong, M.; Oulton, R. F.; Maier, S. A. Multiresonant broadband optical antennas as efficient tunable nanosources of second harmonic light. Nano Lett. 2012, 12, 4997-5002.

(23) Smirnova, D. A.; Khanikaev, A. B.; Smirnov, L. A.; Kivshar, Y. S. Multipolar Third-Harmonic Generation Driven by Optically Induced Magnetic Resonances. ACS Photonics 2016, 3, 1468 1476.

(24) Petschulat, J.; Cialla, D.; Janunts, N.; Rockstuhl, C.; Hübner, U.; Möller, R.; Schneidewind, H.; Mattheis, R.; Popp, J.; Tünnermann, A.; Lederer, F.; Pertsch, T.; Doubly Resonant Optical 
Nanoantenna Arrays for Polarization Resolved Measurements of Surface Enhanced Raman Scattering. Opt. Express 2010, 18, 4184-4197

(25) Linnenbank, H.; Grynko, Y.; Förstner, J.; Linden, S. Second Harmonic Generation Spectroscopy on Hybrid Plasmonic/Dielectric Nanoantennas. Light Sci. Appl. 2016, 5, e16013.

(26) Thyagarajan, K.; Rivier, S.; Lovera, A.; Martin, O. J. F. Enhanced Second-Harmonic Generation from Double Resonant Plasmonic Antennae. Opt. Express 2012, 20, 12860-12865.

(27) Lozovoy, V. V.; Pastirk, I.; Walowicz, K. A.; Dantus, M. Multiphoton Intrapulse Interference. II. Control of Two- and Three-Photon Laser Induced Fluorescence with Shaped Pulses. J. Chem. Phys. 2003, 118, 3187-3196.

(28) Form, N. T.; Whitaker, B. J.; Meier, C. Enhancing the Probability of Three-Photon Absorption in Iodine through Pulse Shaping. J. Phys. B At. Mol. Opt. Phys. 2008, 41, 74011. 